\title{
Uptake and transformation of phenols by duckweed (Lemna gibba)
}

\author{
Takuo FuJISAwA, ${ }^{*}$ Keiko ICHISE-SHiBUYA ${ }^{\dagger}$ and Toshiyuki KatAgI \\ Environmental Health Science Laboratory, Sumitomo Chemical Company, Ltd., \\ 4-2-1 Takatsukasa, Takarazuka, Hyogo 665-8555, Japan \\ † Sumika Technoservice Corporation, 4-2-1 Takatsukasa, Takarazuka, Hyogo 665-8555, Japan
}

(Received May 12, 2010; Accepted July 19, 2010)

\begin{abstract}
Uptake and transformation of phenol and its five derivatives each labeled with ${ }^{14} \mathrm{C}$ were examined in duckweed (Lemna gibba). A kinetic analysis on uptake and metabolic transformation was conducted using the assumed compartments. Positive correlation was observed between $\log \mathrm{P}$ and the logarithm value of relative uptake rate constant with respect to phenol, and an even higher correlation was obtained against the physico-chemical index, EffTox, where the undissociated fraction of phenol was incorporated. No significant correlation was observed between any of the electronic parameters of the phenol derivatives and the transformation rate. These analyses showed that the uptake of phenols by duckweed is mainly controlled by the hydrophobic profile of the undissociated form. For the metabolic profile, the glycoside conjugate was detected as a typical major metabolite for all phenols and the glutathione conjugate as a unique metabolite for 4-chlorophenol. (C) Pesticide Science Society of Japan
\end{abstract}

Keywords: phenol and its derivatives; uptake and transformation; duckweed (Lemna gibba); glucose and glutathione conjugate.

\section{Introduction}

Phenol and its derivatives are common constituents of numerous natural and synthetic chemicals including pesticides. As a consequence of anthropological activities, a portion of them may reach various water bodies as contaminants which will have a great impact on the aquatic environment including biota. Aquatic plants are known to be one of the major habitants and food for many species and hence the distribution and metabolism of chemicals in aquatic plants is very important in order to understand their effects on the aquatic ecosystem. Duckweed is a representative species of aquatic plant, which is widely distributed in freshwater systems. Although the fate of phenol and its derivatives within duckweed has been extensively investigated, ${ }^{1-7)}$ there are few mechanistic studies on the uptake and degradation behavior in relation to their physico-chemical properties. Recently, Tront et al. ${ }^{8)}$ and Reinhold et al. ${ }^{9)}$ studied the uptake of various halogenated phenols into Lemna minor and suggested that the extensive metabolic transformation of phenols via phase II reaction has

\footnotetext{
* To whom correspondence should be addressed.

E-mail: fujisawat1@sc.sumitomo-chem.co.jp

Published online September 6, 2010

(C) Pesticide Science Society of Japan
}

an important role in increasing their uptake by shifting a steady state equilibrium reached between the contaminants in cytosol and medium to a transient state. Tront et al. ${ }^{8)}$ explained that the fraction in the protonated form of chemicals at a cytosolic $\mathrm{pH}$ which was calculated from the corresponding $\mathrm{pKa}$ values had a meaningful correlation with the metabolic transformation rate. In their theory, contaminants that were ionized in the cytosol would exhibit limited partitioning into internal membranes, thereby suppressing the substrate accesses to relevant enzymes. On the other hand, Reinhold et $a l .{ }^{9)}$ reported that the chemical status of phenols within the substrate-enzyme complex is very important when evaluating their transformation rates such as deprotonation of the hydroxy group at the reaction site expressed by their $\mathrm{pKa}$ values and Hammett's constant $(\sigma)$. However, they could not show any direct evidence of a relationship between the uptake and transformation of chemicals because the latter was not actually measured in their studies.

The objective of the present experiment was to examine the uptake and metabolism of phenol and its derivatives by duckweed (Lemna gibba) and to investigate the relationship between the uptake/metabolism behavior of the phenols and various chemical indexes. We also identified some of the phase II metabolites of phenol derivatives produced in duckweed. 


\section{Materials and Methods}

\section{Chemicals}

The ${ }^{14} \mathrm{C}$ materials uniformly labeled at each phenyl ring were used (Fig. 1): phenol (I), 4-nitrophenol (II) and 4-chlorophenol (III) were purchased from Muromachi Pharmaceuticals Co. (Tokyo, Japan); 4-cyanophenol (IV), 3-methyl-4-nitrophenol (V) and 5-methyl-2-nitrophenol (VI) were prepared in our laboratory according to the reported methods. ${ }^{10-12)}$ The specific activities were 31.5 (I), 18.6 (II), 23.0 (III), 16.8 (IV), 29.9 (V) and 13.6 (VI) MBq/mg, with each radiochemical purity being $>97 \%$ as determined by high-performance liquid chromatography (HPLC). Non-radiolabeled authentic standards of I-VI were purchased from Tokyo Chemical Industry Co., Ltd. (Tokyo, Japan). The glucose conjugates of $\mathbf{I V}(\mathbf{X})$ and $\mathbf{V}(\mathbf{X I})$ were synthesized by modifying the procedures reported by Sinnott et al. and Mitts et al., respectively. ${ }^{13,14)}$ Cellulase and $\beta$-glucosidase were purchased from Wako Pure Chemical Industries Ltd. (Osaka, Japan) and Oriental Yeast Co., Ltd. (Tokyo, Japan), respectively.

\section{Spectroscopy}

Liquid chromatography-electrospray ionization-mass spectrometry (LC-ESI-MS) in negative and positive ion modes was performed using a Waters Micromass ZQ spectrometer equipped with Waters Separation Module 2695 and Photodiode Array Detector 2996 as the liquid chromatograph. Samples dissolved in acetonitrile were automatically injected into an ESI source through a Sumipax ODS A-212 column $(150 \mathrm{~mm} \times 6 \mathrm{~mm}$ i.d., $5 \mu \mathrm{m}$, Sumika Chemical Analysis Service Co., Ltd.) at a flow rate of $1.0 \mathrm{ml} \mathrm{min}^{-1}$ using the gradient system with acetonitrile (Solvent A) and $0.1 \%$ formic acid in water (Solvent $\mathrm{B}$ ). The composition of the mobile phase was changed stepwise as follows: $0 \mathrm{~min}, \% \mathrm{~A}-\% \mathrm{~B}, 10-90$; $40 \mathrm{~min}, \% \mathrm{~A}-\% \mathrm{~B}, 90-10(\operatorname{method} 1)$.

\section{Radioassay}

Radioactivity in the plant extracts and exposure water was determined by mixing each aliquot with $10 \mathrm{ml}$ of Packard Scintillator Plus and analyzed by liquid scintillation counting (LSC) with a Packard Model 2900TR spectrometer being

\begin{tabular}{cccc}
\hline Phenol & Conjugate & $\mathrm{R}_{1}, \mathrm{R}_{2}$ \\
\hline $\mathrm{I}$ & $\mathrm{VII}$ & $\mathrm{H}$ & $4-\mathrm{NO}_{2}$ \\
II & $\mathrm{VIII}$ & $4-\mathrm{Cl}$ \\
$\mathrm{III}$ & $\mathrm{IX}$ & $4-\mathrm{CN}$ \\
$\mathrm{IV}$ & $\mathrm{X}$ & $3-\mathrm{CH}_{3}-4-\mathrm{NO}_{2}$ \\
$\mathrm{~V}$ & $\mathrm{XI}$ & $5-\mathrm{CH}_{3}-2-\mathrm{NO}_{2}$ & \\
VI & $\mathrm{XII}$ &
\end{tabular}

Fig. 1. Chemical structures of I-XII. equipped with an automatic external standard. The background level of radioactivity in LSC was $30 \mathrm{dpm}$ on average, which was subtracted from the dpm value of a measured sample. Radioactivity in the unextractable residues of the treated plants was measured using a Packard Model 306 Sample Oxidizer. The unextractable residues were air-dried at room temperature overnight and weighed with a Mettler model AE240, and each aliquot was subjected to combustion. The ${ }^{14} \mathrm{CO}_{2}$ produced was absorbed into $9 \mathrm{ml}$ of Packard Carb- $\mathrm{CO}_{2}$ absorber, mixed with $15 \mathrm{ml}$ of Packard Permafluor scintillator and the radioactivity in it was quantified by LSC. The efficiency of combustion was determined to be greater than $90 \%$.

\section{Chromatographies}

High performance liquid chromatography (HPLC) was conducted by using a Hitachi L-7100 Pump linked in series with a L-7405 UV detector and a Packard Radiomatic 505TR radio detector equipped with a 500- $\mu 1$ liquid cell. Ultima-Flo AP (Packard Instrument Co.) was utilized as the scintillator. A Sumipax ODS A-212 column $(150 \mathrm{~mm} \times 6 \mathrm{~mm}$ i.d., $5 \mu \mathrm{m})$ was employed for both analytical and preparative purposes at a flow rate of $1 \mathrm{ml} \mathrm{min}^{-1}$. The following gradient system was used for typical analysis of metabolites with $0.01 \%$ trifluoroacetic acid (Solvent A) and acetonitrile (Solvent B): 0 min, $\% \mathrm{~A}-\% \mathrm{~B}, 90-10 ; 40 \mathrm{~min}, \% \mathrm{~A}-\% \mathrm{~B}, 10-90 ; 40.1 \mathrm{~min}, \% \mathrm{~A}-\% \mathrm{~B}$, 0-100; $50 \mathrm{~min}, \% \mathrm{~A}-\% \mathrm{~B}, 0-100$ (method 2). Retention times of $\mathbf{I}-\mathbf{V I}, \mathbf{X}$ and $\mathbf{X I}$ are 16.6, 20.1, 23.3, 16.9, 27.4, 22.1, 9.2 and $13.6 \mathrm{~min}$, respectively.

Thin-layer chromatography (TLC) was conducted using silica gel $60 \mathrm{~F}_{254}$ thin-layer chromatoplates $(20 \times 20 \mathrm{~cm}, 0.25-\mathrm{mm}$ thickness, E. Merck) for glucose conjugates of IV (X) and $\mathbf{V}$ (XI). For the solvent system for development, toluene/ethyl formate/formic acid, 5/7/1 (v/v/v) was used. The non-radiolabeled reference standards were detected by exposing TLC plates to ultraviolet light. Autoradiograms were prepared by exposing TLC plates to a BAS-IIIs Fuji Imaging Plate for several hours. The radioactivity on an imaging plate was detected by using an Amersham Variable Mode Imager Typhoon 9200. The $R_{\mathrm{f}}$ values of glucose conjugates of IV (X) and $\mathbf{V}$ (XI) were 0.03 and 0.08 , respectively.

\section{Plant material, maintenance and treatment}

Duckweed (Lemna gibba) was obtained from an agricultural waterway located at Naruto-city (Tokushima, Japan). The duckweed plants were maintained in pots filled with a water/sediment prepared by mixing tap water and a Kasai clay loam soil in a greenhouse at $25^{\circ} \mathrm{C}$. Prior to the experiments, the duckweed was removed from the pot, thoroughly washed with distilled water and sterilized with $0.5 \%$ sodium hypochloride for $10 \mathrm{~min} .{ }^{15)}$ The acetonitrile dose solution of ${ }^{14} \mathrm{C}$ test substances was individually prepared by isotopically diluting them with the corresponding non-radiolabeled reference standards to give the specific activity of $0.49 \mathrm{MBq} / \mathrm{mg}$. A $20 \mu \mathrm{l}$ of the dose solution was spiked into $100 \mathrm{ml}$ of 
Hoagland's medium at $\mathrm{pH} 7.0^{16)}$ in a $100-\mathrm{ml}$ cylindrical glass vessel which produced a final concentration of $0.1 \mu \mathrm{g} \mathrm{ml}^{-1}$ and total radioactivity of $50 \mathrm{kBq}$. The sterile plants with a fresh weight of $5 \mathrm{~g}$ were added to the glass vessel which was then covered with a Pyrex plate sealed by Parafilm. Twelve sealed glass vessels per test substance were then maintained in a climate chamber Biotron LPH-200 (Nippon Medical \& Chemical Instruments Co., Ltd.) with $24 \mathrm{hr}$ irradiation at $12,500 \mathrm{~lx}$ and $25^{\circ} \mathrm{C}$, and the plants and exposure water were sampled in duplicate at $1,2,4,8,16$ and $24 \mathrm{hr}$. To collect sufficient amounts of unknown metabolites IX and XIII for spectrometric analyses, $100 \mathrm{ml}$ of aqueous solutions of $\left[{ }^{14} \mathrm{C}\right]-$ III at the concentration of $1 \mu \mathrm{g} \mathrm{ml}^{-1}$ were separately prepared in a $200-\mathrm{ml}$ beaker and plants were exposed to the medium for 4 days.

\section{Extraction of plants and isolation of metabolites}

Duckweed was separated from the exposure water by filtering under a vacuum. Aliquots of the exposure water were individually radioassayed by LSC. Duckweed was extracted using an AM-8 Ace homogenizer (Nihon Seiki Co., Ltd.) at 14,000 $\mathrm{rpm}$ for $10 \mathrm{~min}$ with acetone/water $(4 / 1, \mathrm{v} / \mathrm{v})$ at the ratio of 5 $\mathrm{ml}$ per one gram of plant. The mixture was filtered under suction and the residue was extracted two additional times in the same way. Aliquots of plant extracts were radioassayed by LSC and then concentrated to dryness in vacuo, re-dissolved in a small volume of organic solvent, and each aliquot was subjected to HPLC and TLC analyses. Unextractable residues were air-dried in open vessels at room temperature overnight and sub-samples of the dried residues were subjected to combustion analysis to determine the remaining radioactivity.

\section{Identification of metabolites}

Identification of metabolites was first conducted by HPLC and TLC co-chromatographies with non-radiolabeled reference standards. The metabolites that did not match with any reference standards by simple co-chromatography were subjected to LC-MS analysis and/or enzymatic hydrolysis. Plant extract including 20,000 dpm was evaporated to dryness and re-dissolved in $1 \mathrm{ml}$ of $10 \mathrm{mM}$ phosphate buffer at $\mathrm{pH}$ 5. Suc-

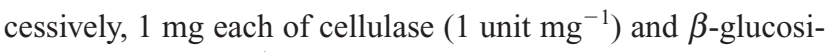
dase ( 37 units $\mathrm{mg}^{-1}$ ) was added to the solution and incubated overnight at $37^{\circ} \mathrm{C} .^{17)}$

\section{Calculations}

In order to examine the behavior of phenols, kinetic analysis based on the assumed compartments was conducted using the Model-Maker program (version 4, ModelKinetix). The relative rate constants on uptake and degradation for substituted phenols with respect to phenol were obtained and their logarithm values were subjected to regression analysis with various chemical indexes using Microsoft Excel 2003.

The physico-chemical parameters used for analysis are listed in Table 1. The fraction of the undissociated form of each phenol in the medium at $\mathrm{pH} 7.0\left(f_{\text {medium }}\right)$ was calculated from their acid dissociation constant $\left(K_{\mathrm{a}}\right)$ by the following equation.

$$
f_{\text {medium }}=\left(K_{\mathrm{a}} / 10^{-7}+1\right)^{-1}
$$

In the U.S. EPA AQUATOX model, ${ }^{18)}$ a piece-wise theory is applied to evaluate the uptake of an ionizable toxicant through a lipid membrane and the EffTox index is introduced for chemicals having $\log \mathrm{P}$ of $1.5-3.0$ as follows.

$$
\text { EffTox }=0.1+f_{\text {medium }} \times(0.3 \times \log \mathrm{P}-0.45)
$$

Since glucosylation is considered to proceed via attack of the phenolic oxygen on the $\mathrm{C} 1$ position of a glucose moiety being catalyzed by glucosyltransferase, ${ }^{19)}$ the electron distribution on the phenolic oxygen atom in the highest occupied molecular orbital (HOMO) becomes a potential index to explain the conjugation rate. The molecular geometry of each phenol and the corresponding phenolate ion generated from the standard bond lengths and angles were fully optimized by MNDO-PM3 in the WINMOPAC program (version 3.9, Fujitsu Ltd.). The electrophilic reaction index $\left(f_{\mathrm{E}(\mathrm{OH})}\right.$ and $\left.f_{\mathrm{E}\left(\mathrm{O}^{-}\right)}\right)$ for each species was then calculated from the following equation.

$$
f_{\mathrm{E}}=2 \sum_{i} \mathrm{C}_{\mathrm{HOMO}}^{2} \quad\left(i=s, \mathrm{p}_{\mathrm{x}}, \mathrm{p}_{\mathrm{y}}, \mathrm{p}_{\mathrm{z}}\right)
$$

\begin{tabular}{|c|c|c|c|c|c|c|c|c|}
\hline Compound & $\log \mathrm{P}^{a)}$ & $\mathrm{p} K \mathrm{a}^{b)}$ & $f_{\text {medium }}{ }^{c)}$ & EffTox ${ }^{d)}$ & $\sigma$ & $\sigma^{-}$ & $f_{\mathrm{E}(\mathrm{OH})^{e}}$ & $f_{\mathrm{E}\left(\mathrm{O}^{-}\right)}{ }^{e)}$ \\
\hline I & 1.46 & 9.98 & 0.9990 & 0.1 & 0 & 0 & 0.1476 & 0.2136 \\
\hline II & 1.91 & 7.15 & 0.5855 & 0.1720 & 0.78 & 1.24 & $5.3 \times 10^{-5}$ & 0.0274 \\
\hline III & 2.39 & 9.38 & 0.9961 & 0.3660 & 0.23 & 0.27 & 0.0998 & 0.1954 \\
\hline IV & 1.60 & 7.97 & 0.9032 & 0.1270 & 0.66 & 1 & $1.0 \times 10^{-6}$ & 0.1714 \\
\hline $\mathbf{V}$ & 2.12 & 7.33 & 0.6813 & 0.2820 & 0.71 & 0.71 & $4.7 \times 10^{-4}$ & 0.0271 \\
\hline VI & 2.31 & 7.41 & 0.7200 & 0.2749 & 0.73 & 0.73 & 0.0305 & 0.0232 \\
\hline
\end{tabular}

Table 1. The physico-chemical parameters of I-VI.

${ }^{a)}$ Experimental values from Ref 33-36. ${ }^{b)}$ Experimental values from Ref 37. ${ }^{c)} f_{\text {medium }}=\left(K_{\mathrm{a}} / 10^{-7}+1\right)^{-1}$ d) $E f f T o x=0.1+f_{\text {medium }} \times(0.3 \times \log \mathrm{P}-0.45)$.

e) The electrophilic reaction index of the phenolic oxygen of undissociated $\left[f_{\mathrm{E}(\mathrm{OH})}\right]$ and dissociated $\left[f_{\mathrm{E}\left(\mathrm{O}^{-}\right)}\right]$forms. 
$\mathrm{C}_{\text {номо }}$ means the coefficient of each atomic orbital $\left(\mathrm{s}, \mathrm{p}_{\mathrm{x}}, \mathrm{p}_{\mathrm{y}}\right.$ and $\left.\mathrm{p}_{\mathrm{z}}\right)$ in HOMO. The Hammett's constants $\left(\sigma\right.$ and $\left.\sigma^{-}\right)$were calculated with the established methodology of Hansch and Leo $^{20)}$ and Fujita. ${ }^{21)}$

\section{Results}

1. ${ }^{14} \mathrm{C}$ Distribution in the test system

Each phenol was gradually taken up by duckweed with its extent dependent on the chemical structures, as shown in Fig. 2. The ${ }^{14} \mathrm{C}$ distribution in the test system after $24 \mathrm{hr}$ is summarized in Table 2. The recovered ${ }^{14} \mathrm{C}$ from the test systems during the exposure period was $90.0-105.6 \%$ of the applied ${ }^{14} \mathrm{C}$ (AR) for I-V, but it decreased to $82.6 \%$ AR for VI probably due to its azeotropic vaporization. The amount of uptake after $24 \mathrm{hr}$ was largest for III (38.4\% AR) and smallest for IV $(15.0 \%$ AR). Most of the radioactivity was extracted from duckweed except for III which had significant unextractable (bound) residues (12.2\% AR).

\section{Distribution of metabolites}

The metabolic distribution in duckweed at 24-hr exposure is summarized in Table 2. No degradation of $\mathbf{I}-\mathbf{V I}$ in the growth medium was observed under the test conditions, indicating insignificant contributions of photolysis and microbial degradation. The glycoside conjugate of each phenol was the main metabolite and the glutathione conjugate (XIII) was uniquely produced from III at the level of $12.6 \% \mathrm{AR}$.

The glycoside conjugates (VII, VIII and XII) were firstly subjected to enzymatic hydrolysis using cellulase and $\beta$-glucosidase which quantitatively produced the corresponding phenols (I, II and VI) as aglycone. The chemical structure of each aglycone was confirmed by HPLC co-chromatography with the corresponding reference standard. Furthermore, LC-
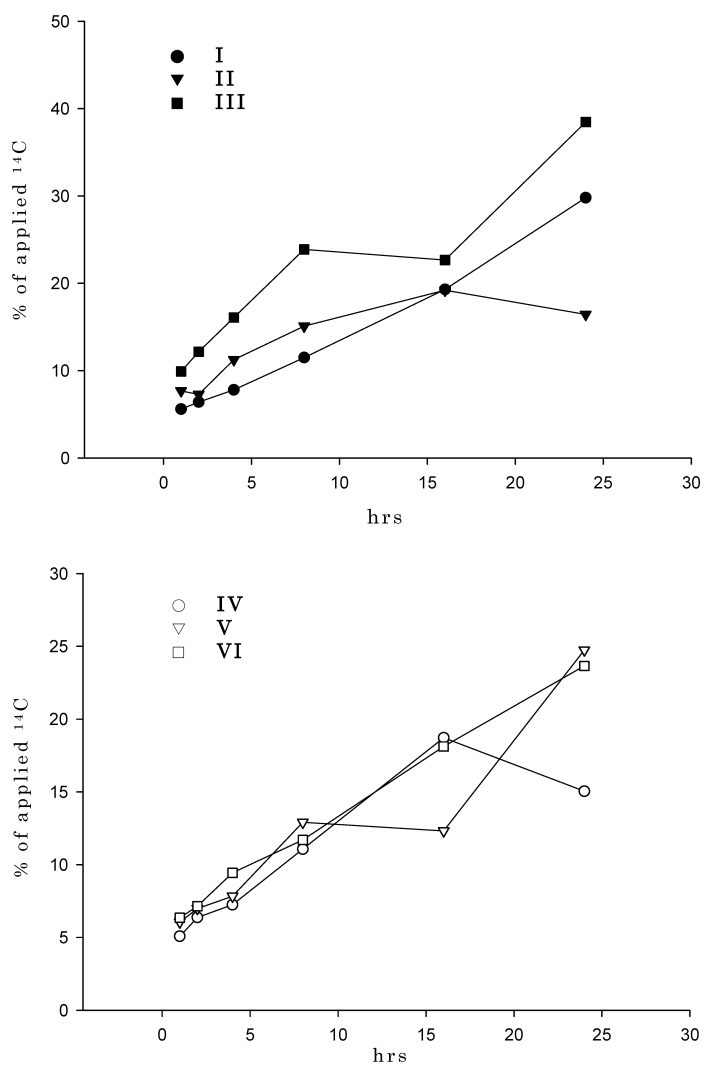

Fig. 2. Radioactivity taken up by duckweed.

MS spectra of each conjugate showed the pseudo-ion $[\mathrm{M}-\mathrm{H}]^{-}$at $\mathrm{m} / \mathrm{z} 255$ (VII), 300 (VIII) and 314 (XII) clearly indicating the mono-saccharide form. The glucoside conjugates $\mathbf{X}$ and $\mathbf{X I}$ were confirmed by the HPLC and TLC cochromatographies using their reference standards. The un-

Table 2. ${ }^{14} \mathrm{C}$ distribution of I-VI at 24-hr exposure.

\begin{tabular}{|c|c|c|c|c|c|c|}
\hline & \multicolumn{6}{|c|}{$\%$ of the applied ${ }^{14} \mathrm{C}$} \\
\hline & $\mathbf{I}$ & II & III & IV & $\mathbf{V}$ & VI \\
\hline Duckweed ${ }^{14} \mathrm{C}$ & 21.7 & 16.4 & 38.4 & 15.0 & 24.8 & 23.7 \\
\hline Extractable & 16.3 & 15.1 & 26.2 & 14.4 & 23.0 & 20.5 \\
\hline phenol & 0.5 & 8.8 & 4.3 & 6.6 & 13.3 & 5.6 \\
\hline glucose-conj. & 15.4 (VII) & $5.2(\mathrm{VIII})$ & $6.7(\mathbf{I X})$ & $7.7(\mathbf{X})$ & 9.7 (XI) & $13.4(\mathbf{X I I})$ \\
\hline glutathione-conj. & nd & nd & 12.6 (XIII) & nd & nd & nd \\
\hline others & 0.5 & 1.1 & 2.7 & nd & nd & 1.6 \\
\hline Bound & 5.4 & 1.3 & 12.2 & 0.6 & 1.8 & 3.2 \\
\hline Medium ${ }^{14} \mathrm{C}$ & 68.3 & 82.1 & 59.9 & 84.2 & 80.8 & 58.9 \\
\hline phenol & 68.3 & 82.1 & 59.9 & 84.2 & 80.8 & 58.9 \\
\hline others & nd & nd & nd & nd & nd & nd \\
\hline Total ${ }^{14} \mathrm{C}$ & 90.0 & 98.5 & 98.3 & 99.3 & 105.6 & 82.6 \\
\hline
\end{tabular}

nd: not detected. 

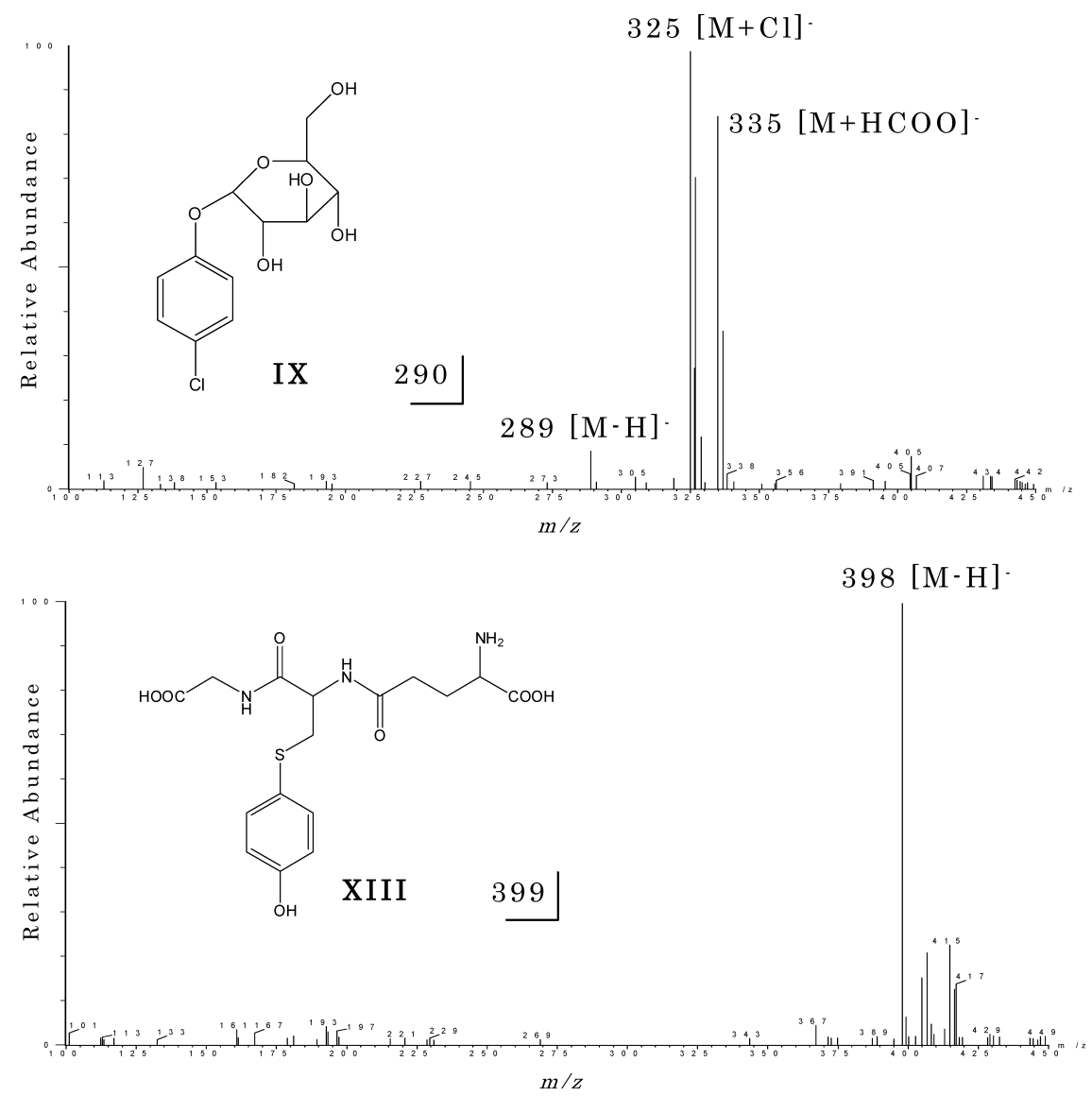

Fig. 3. LC-MS spectra of IX and XIII.

known metabolites IX and XIII originating from III were isolated from the plant extracts and their molecular weights were estimated to be 290 and 399, respectively, by LC-ESI-MS analysis in the negative ion mode (Fig. 3). The pseudo-ions of IX were observed at $m / z 289[\mathrm{M}-\mathrm{H}]^{-}, 325[\mathrm{M}+\mathrm{Cl}]^{-}$and $335\left[\mathrm{M}+\mathrm{HCOO}^{-}\right.$with its daughter ion at $\mathrm{m} / z 127$ [M-glucose $-\mathrm{H}]^{-}$, which suggested its chemical structure to be the

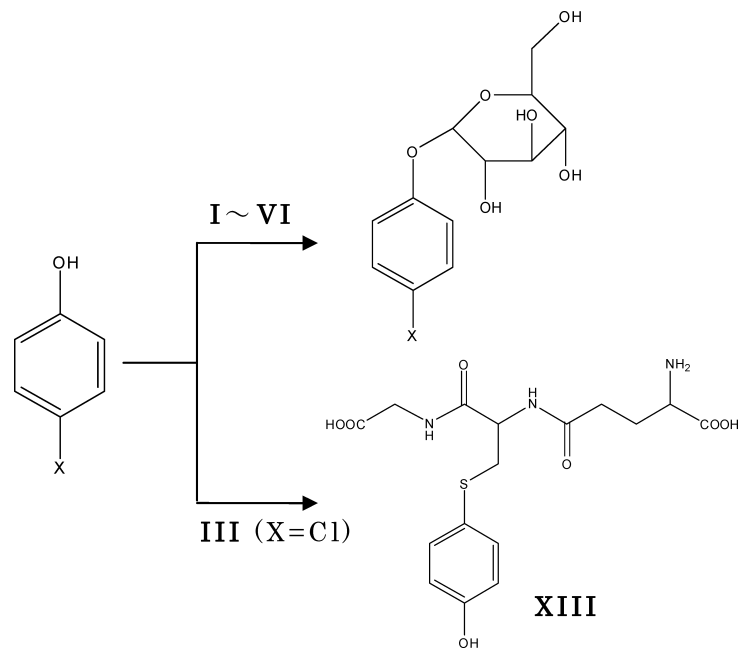

Fig. 4. Proposed metabolic pathways of phenols in duckweed. mono-glycoside conjugate of III. The chemical structure of the aglycone via enzymatic hydrolysis was also confirmed by HPLC and TLC co-chromatographies with the reference standard. Incidentally, the LC-ESI-MS spectrum of XIII in the negative ion mode showed an ion peak at $\mathrm{m} / \mathrm{z} 398$. The molecular weight of 399 together with no isotopic ion pattern unique to a chlorine atom strongly suggested XIII to be the glutathione (GSH) conjugate of III. III was considered to be conjugated with GSH, a high molecular natural component existing in lemna, ${ }^{22)}$ via substitution reaction of chlorine at 4position on the aromatic ring with the sulfur atom of GSH. The metabolic pathways of the phenols tested are summarized in Fig. 4.

\section{Kinetic analysis}

A simple four- or five-compartment model as shown in Fig. 5 was utilized for the kinetic analysis. With regard to duckweed, each compartment was assigned to the major conjugates and bound ${ }^{14} \mathrm{C}$. Since the amount of unknown metabolites was insignificant, it was conveniently incorporated into the bound compartment. Each rate constant was optimized as each curve was well fitted to the observed data, as shown in Fig. 6. The results of rate constant with their coefficients of correlation are listed in Table 3 . The correlation of $\log \left(k_{l(\mathrm{i})} / k_{l(\mathbf{I})}\right)$, the log- 


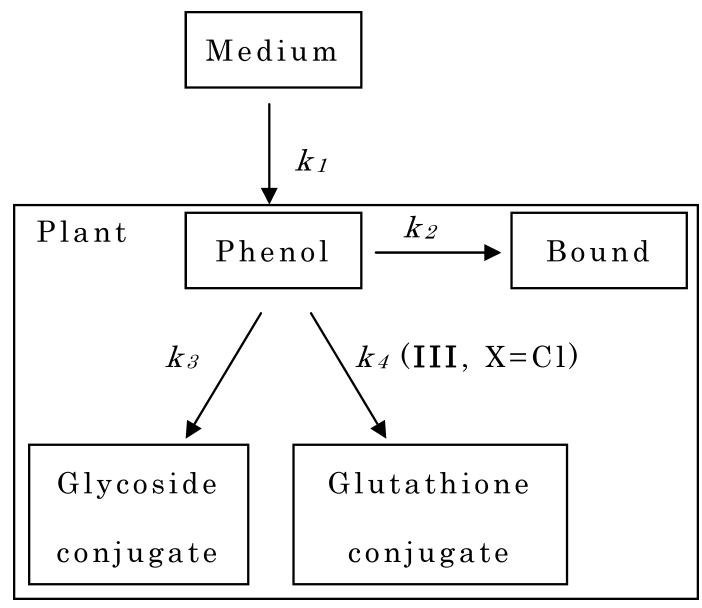

Fig. 5. Compartment model used for kinetic analysis.

arithm value of the relative uptake rate constant of phenol derivatives with respect to $\mathbf{I}$, with $\log \mathrm{P}$ was moderate, but the usage of the EffTox index instead improved the correlation as shown in Table 4.

$$
\begin{aligned}
& \log \left(k_{l(\mathrm{i})} / k_{l(\mathbf{I})}\right)=0.505 \log \mathrm{P}-0.0136 \quad(r=0.554, s=0.321, n=6) \\
& \log \left(k_{l(\mathrm{i})} / k_{l(\mathbf{I})}\right)=2.681 \text { EffTox }-0.413 \quad(r=0.761, s=0.250, n=6)
\end{aligned}
$$

For the degradation rate constant of glucose conjugate, the correlation of logarithm values expressed as $\log \left(k_{3(\mathrm{i})} / k_{3(\mathbf{I})}\right)$ and various chemical parameters such as $\sigma, \sigma^{-}, f_{\mathrm{E}(\mathrm{OH})}$ and $f_{\mathrm{E}\left(\mathrm{O}^{-}\right)}$ were calculated as $0.271,0.261,-0.239$ and -0.259 , respectively, which showed no conspicuous trend. Additionally, direct comparison between $\log \left(k_{l(\mathrm{i})} / k_{l(\mathbf{I})}\right)$ and $\log \left(k_{3(\mathrm{i})} / k_{3(\mathbf{I})}\right)$ values showed a good correlation of -0.700 . The production rate of the GSH conjugate for III was taken into account to obtain the correlation between $\log \left(k_{l(\mathrm{i})} / k_{l(\mathrm{I})}\right)$ and that for total transformation rate of conjugates using $\log \left(\left(k_{3 \text { (III) }}+k_{4(\text { III })}\right) /\right.$ $\left.k_{3(\mathbf{I})}\right)$ instead of $\log \left(k_{3(\mathrm{III})} / k_{3(\mathbf{I})}\right)$, which was calculated to be -0.270 .

\section{Discussion}

The usage of the EffTox index expressing the uptake of a partially ionized chemical gave a better correlation with the log $\left(k_{l(\mathrm{i})} / k_{l(\mathbf{I})}\right)$ than the simple $\log \mathrm{P}$ value, which clearly demonstrated the significant involvement of the undissociated species of each phenol in its uptake into duckweed. A similar theory has been proposed for terrestrial plants. For example, Briggs et al. ${ }^{23)}$ clarified that the translocation from root to shoot vs. $\log \mathrm{P}$ plot showed Gaussian curves with maximum peaks observed at $\log \mathrm{P}$ of 2 to 3 for undissociated chemicals, but their uptake drastically decreased in accordance with the increasing degree of ionization. Smejtek et al. ${ }^{24)}$ reported that it was difficult for ionized species to penetrate lipid membranes via electrostatic repulsion between the adsorbed and free species, which results in less partition. This concept agrees well with the definition of EffTox and explains the largest uptake by duckweed for III which is most hydrophobic and almost all present as an undissociated form at the tested $\mathrm{pH}$. Secondly, we examined whether or not there were any correlations between the degradation of phenols and electronic parameters $\left(\sigma, \sigma^{-}, f_{\mathrm{E}(\mathrm{OH})}\right.$ and $\left.f_{\mathrm{E}\left(\mathrm{O}^{-}\right)}\right)$, but no significant correlation was observed. We had expected to find some trend between the $\log \left(k_{3(\mathrm{i})} / k_{3(\mathrm{I})}\right)$ value and electronic parameters because the latter are considered to be important parameters expressing the availability of an easily deprotonated hydroxy group. This deprotonation of the hydroxy group is a prerequisite reaction for the transfer of glucose to it via glucosyltransferase. $^{8,9)}$ In this respect, Shao et al. ${ }^{25)}$ and Achnine et al. ${ }^{26)}$ reported on a molecular docking analysis of the interaction between glycosyltransferase and the donor ligand flavonoid.

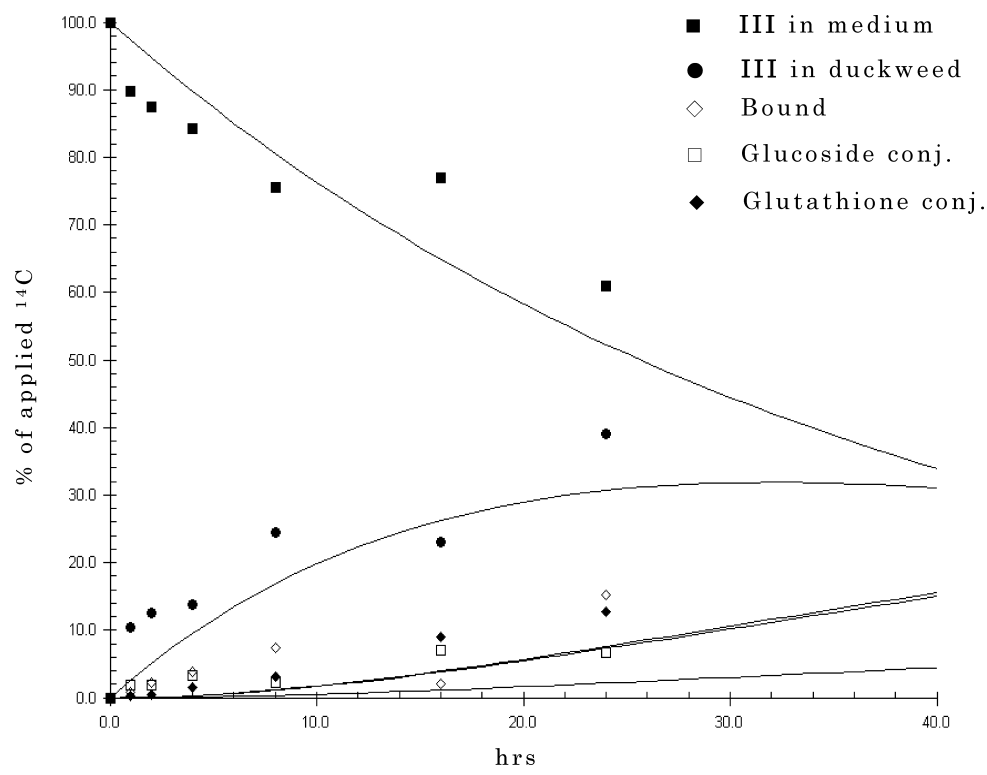

Fig. 6. Uptake and metabolic profiles of III simulated with the Model-Maker program in duckweed. 
Table 3. Kinetic analysis of the degradation of $\mathbf{I}-\mathbf{V I}$.

\begin{tabular}{|c|c|c|c|c|c|c|}
\hline $\begin{array}{l}\text { Rate constant } \\
\qquad\left(\mathrm{hrs}^{-1}\right)\end{array}$ & $\mathbf{I}$ & II & III & IV & $\mathbf{V}$ & VI \\
\hline$k_{l}$ (uptake) & 0.0167 & 0.0131 & 0.0270 & 0.0114 & 0.0126 & 0.0186 \\
\hline$k_{2}$ (bound) & 0.0081 & 0.0049 & 0.0152 & $1.0 \times 10^{-7}$ & $1.0 \times 10^{-7}$ & $1.0 \times 10^{-7}$ \\
\hline$k_{3}$ (glucose) & 0.0410 & 0.0249 & 0.0045 & 0.0370 & 0.0232 & 0.0377 \\
\hline$k_{4}(\mathrm{GSH})$ & - & - & 0.0158 & - & - & - \\
\hline$r^{2}$ & 0.9898 & 0.9884 & 0.9735 & 0.9937 & 0.9948 & 0.9910 \\
\hline \multicolumn{7}{|l|}{ Metabolic transformation } \\
\hline $\log \left(k_{I(\mathrm{i})} / k_{I(\mathbf{I})}\right)^{a)}$ & 0 & -0.1078 & 0.2069 & -0.1666 & -0.1226 & 0.0461 \\
\hline $\log \left(k_{3(\mathrm{i})} / k_{3(\mathbf{I})}\right)^{a)}$ & 0 & -0.2166 & -0.9596 & -0.0438 & -0.2473 & -0.0369 \\
\hline $\log \left(\left(k_{3(\mathrm{III})}+k_{4(\mathrm{III})}\right) / k_{3(\mathrm{I})}\right)$ & - & - & -0.3050 & - & - & - \\
\hline
\end{tabular}

The sequence alignment of the peptide in the active site forms an electron transfer chain which promotes deprotonation of the hydroxy group, enhancing the conjugation with monosaccharide. Additionally, the electronically repelling force produced due to the spatial arrangement of compound in the active site prevents the smooth progress of glycosylation. These facts clearly indicate that the precise evaluation of the electrostatus of the chemicals within the substrate-enzyme complex under a steric constraint may become very important to estimate the degradation behavior in duckweed. We incidentally found a positive trend between the degradation of the compounds via glycosyltransferase and the uptake of chemicals into duckweed as suggested by Tront et al. ${ }^{8)}$ and Reinhold et $a l .{ }^{9}{ }^{9}$ which was evaluated by direct comparison of the $\log$ $\left(k_{l(\mathrm{i})} / k_{l(\mathbf{I})}\right)$ and $\log \left(k_{3(\mathrm{i})} / k_{3(\mathbf{I})}\right)$ values. However, less correlation was observed between the logarithms of uptake and transformation when the production rate to form glutathione conjugate of III was reflected to the parameter. This implies that further research is still required to clarify their precise relationships because the glutathione conjugation of xeonobiotics is well known to be a prerequisite reaction for their efficient transfer to the vacuole as it is described with glycosylation. ${ }^{27)}$

The major secondary metabolite observed in duckweed exposed to I-VI was the glycoside conjugates. Glycoside conjugation is known to be one of the most common transformations for chemicals having the amino, hydroxy or carboxy group in plants. ${ }^{28)}$ The metabolic fate of xenobiotics in duckweed has been studied in the past and the glycoside conju- gates of the corresponding phenols was clarified as the major metabolites. $^{1-7)}$ Days and Saunders ${ }^{29)}$ reported different conjugations such as malonyl glucoside and apiose which enhance the transportation of the resultant conjugates to vacuole and cell-wall, respectively. By the way, it is widely known that, in the plant kingdom, glutathione $S$-transferase plays an important role in the protection of cells from various endogenous and exogenous xenobiotics. Roy et l. $^{30)}$ and Schrenk et al. ${ }^{31)}$ reported the existence of glutathione $S$-transferase in aquaticmacrophytes and confirmed the elevation of the enzyme after exposure to chemicals followed by the formation of the corresponding polar degradates. Although the chemical structures of GSH conjugates have been identified by LC-MS for herbicide isoproturon in aquatic plants, ${ }^{32)}$ there are few studies reporting the exact chemical structure of the GSH conjugate produced by duckweed. In this respect, we conducted an LCESI-MS spectrometric analysis and clearly identified the chemical structure of the glutathione conjugate of III (XIII).

In conclusion, we have clarified that the uptake of chemicals into duckweed shows a positive relationship with the log $\mathrm{P}$ value, and this was further improved by reflecting the ionized ratio of the compounds in the surrounding medium into the parameter, EffTox. For the metabolic transformation of chemicals, some meaningful trend was observed between the degradation rate of chemicals by glycosyltransferease and their uptake rate into duckweed, but no other specific relationship was detected with any electronic parameters of the phenol derivatives. With regard to metabolism, all phenols under-

Table 4. The correlation between logarithm values of the relative rate constant and various parameters of phenol derivatives.

\begin{tabular}{rrrrrrrrr}
\hline & \multicolumn{1}{c}{$\log \mathrm{P}$} & $\mathrm{pKa}$ & $f_{\text {medium }}$ & EffTox & \multicolumn{1}{c}{$\sigma$} & $\sigma^{-}$ & $f_{\mathrm{E}(\mathrm{OH})}$ & $f_{\mathrm{E}\left(\mathrm{O}^{-}\right)}$ \\
\hline $\log \left(k_{l(\mathrm{i})} / k_{l(\mathrm{I})}\right)$ & 0.579 & 0.565 & 0.489 & 0.640 & -0.560 & -0.641 & 0.659 & 0.350 \\
$\log \left(k_{3(\mathrm{i})} / k_{3(\mathrm{I})}\right)$ & -0.630 & -0.299 & -0.291 & -0.761 & 0.271 & 0.261 & -0.239 & -0.259 \\
\hline
\end{tabular}


went phase II reaction with the natural components such as monosaccharide and glutathione which may be the prerequisite process for the precise transportation of the resultant compounds to the designated intracellular and intercellular organelles.

\section{References}

1) H. E. Ensley, H. A. Sharma, J. T. Barber and M. A. Polito: "Metabolism of Chlorinated Phenols by Lemna gibba, Duckweed," ed. by E. L. Kruger, T. A. Anderson and J. R. Coats, ACS Symp. Ser. 664, American Chemical Society, Washington, D.C., Chap. 17, pp. 238-253, 1997.

2) J. T. Barber, H. A. Sharma, H. E. Ensley, M. A. Polito and D. A. Thomas: Chemosphere 31, 3567-3574 (1995).

3) H. E. Ensley, J. T. Barber, M. A. Polito and A. I. Oliver: Environ. Toxicol. Chem. 13, 325-331 (1994).

4) S. Pascal-Lorber, E. Rathahao, J.-P. Cravedi and F. Laurent: Chemosphere 56, 275-284 (2004).

5) V. Ugrekhelidze: J. Bio. Phys. Chem. 2, 38-41 (2002).

6) J. M. Tront and F. M. Saunders: Chemosphere 64, 400-407 (2006).

7) T. Fujisawa, M. Kurosawa and T. Katagi: J. Agric. Food Chem. 54, 6286-6293 (2006).

8) J. M. Tront, D. M. Reinhold, A. W. Bragg and F. M. Saunders: $J$. Environ. Eng. 133, 955-961 (2007).

9) D. M. Reinhold and F. M. Saunders: Trans. ASABE. 49, 2077-2083 (2006).

10) A. Yoshitake, K. Kawahara, T. Kamada and M. Endo: J. Labelled Compd. Radiopharm. 13, 323-331 (1977).

11) A. Yoshitake, F. Shono, T. Kamada and I. Nakatsuka: J. Labelled Compd. Radiopharm. 13, 333-338 (1977).

12) A. Yoshitake, H. Kanamaru, F. Shono and I. Nakatsuka: J. Labelled Compd. Radiopharm. 16, 477-482 (1979).

13) M. L. Sinnot and I. J. L. Souchard: Biochem. J. 133, 89-98 (1973).

14) E. Mitts and R. M. Hixon: J. Am. Chem. Soc. 66, 483-486 (1944).

15) E. Landolt and R. Kandeln: "The Family of Lemnaceae: A monographic study, Vol. 2", Geobotanischen Institutes der ETH, Stiftung Rubel: Zurich, Switzerland, p. 638, 1987.

16) C. F. Cleland and W. R. Briggs: Plant Physiol. 42, 1553-1561 (1967).

17) T. Shishido and H. Ohkawa: "Methods in Pesticide Science," ed. by J. Fukami, Y. Uesugi, K. Ishizuka and C. Tomizawa, Soft Science, Tokyo, Chap. 1, pp. 3-67, 1981 (in Japanese).

18) J. M. McKim, P. Schmeider and G. Veith: Tox. App. Pharm. 77,
1-10 (1985).

19) W. Offen, C. Martinez-Fleites, M. Yang, E. Kiat-Lim, B. G. Davis, C. A. Tarling, C. M. Ford, D. J. Bowles and G. J. Davies: EMBO J. 25, 1396-1405 (2006).

20) C. Hansch and A. Leo: "Exploring QSAR: Fundamentals and applications in Chemistry and Biology," American Chemical Society, Washington, D.C., 1995.

21) T. Fujita: "Structure-Activity Relationships-Quantitative Approaches; Applications to Drug Design and Mode-of-Action Studies," ed. by T. Fujita, Nankodo, Tokyo, 1982.

22) S. Pflugmacher, P. Schröder and H. Sandermann Jr.: Phytochem. 54, 267-273 (2000).

23) G. G. Briggs, R. H. Bromilow and A. A. Evans: Pestic. Sci. 13, 495-504 (1982).

24) P. Smejtek and S. Wang: Arch. Environ. Contam. Toxicol. 25, 394-404 (1993).

25) H. Shao, X. He, L. Achnine, J. W. Blount, R. A. Dixon and X. Wang: Plant Cell 17, 3141-3154 (2005).

26) L. Achnine, D. V. Huhman, M. A. Farag, L. W. Sumner, J. W. Blount and R. A. Dixon: Plant J. 41, 875-887 (2005).

27) D. M. Bartholomew, D. E. van Dyk, S. C. Lau, D. P. O'Keefe and P. A. Rea: Plant Physiol. 130, 1562-1572 (2002).

28) G. L. Lamoureux and D. G. Rusness: "Xenobiotic Conjugation in Higher Plants" ed. by G. D. Paulson, J. Cardwell, D. H. Hutson and J. J. Menn, ACS Symp. Ser. 299, American Chemical Society, Washington, D.C., Chap. 4, pp. 62-105, 1986.

29) J. A. Days and F. M. Saunders: Environ. Toxicol. Chem. 23, 613-620 (2004).

30) S. Roy and O. Hänninen: Environ. Toxicol. Chem. 13, 763-773 (1994).

31) C. Schrenk, S. Pflugmacher, R. Brugemann. H., Jr. Sandermann and C. E. W. Steinberg and A. Kettrup: Ecotoxicol. Environ. Saf. 40, 226-233 (1998).

32) C. Pietsch, E. Krause, B. K. Burnison and C. E. W. Steinberg: J. Appl. Bot. Food Qual. 80, 25-30 (2006).

33) R. P. Schwarzenbach, R. Stierli, B. R. Folsom and J. Zeyer: Environ. Sci. Technol. 22, 83-92 (1988).

34) X. Liu, J. Chen, H. Yu, J. Zhao, J. P. Giesy and X. Wang: Chemosphere 64, 1619-1626 (2006).

35) S. Taj, S. Sankarapapavinsam and M. F. Armed: Synth. Met. 97, 205-209 (1998).

36) OECD SID Initial Assessment Report, 3-Methyl-4-nitro-phenol (1994).

37) B. G. Tehan, E. J. Lloyd, M. G. Wong, W. R. Pitt, J. G. Montana and E. Gancia: Quant. Struc.-Act. Relat. 21, 457-472 (2002). 